Research Article

\title{
Compound Grey-Logistic Model and Its Application
}

\author{
Xiao-Lan Wu, Sheng-Yuan Wang $(D$, and Guo-Yin Xu \\ Nanjing XiaoZhuang University, Nanjing, Jiangsu 211171, China \\ Correspondence should be addressed to Sheng-Yuan Wang; 56439976@qq.com \\ Received 5 April 2021; Revised 26 April 2021; Accepted 21 May 2021; Published 27 May 2021 \\ Academic Editor: Alessandro Contento \\ Copyright $\odot 2021$ Xiao-Lan Wu et al. This is an open access article distributed under the Creative Commons Attribution License, \\ which permits unrestricted use, distribution, and reproduction in any medium, provided the original work is properly cited. \\ Logistic regression model is widely used in ecology and in the analysis of social economic systems, because of its good adaptability. \\ In order to improve the measurement accuracy of logistic model, this paper proposes a new method. A compound grey-logistic \\ model is developed to carry out the grey transformation of the original data. Practice shows that the grey transformation data has \\ better simulation accuracy; at the same time, grey transformation can reduce the observation noise of the original data. Mean \\ absolute percentage error index has been used to evaluate the accuracy of prediction model, and information entropy can be used \\ to evaluate the change of information entropy of forecasting data. In this paper, three cases are used to verify the applicability of \\ grey-logistic model. From the perspective of the type of original data, the three cases represent three different data conditions: \\ sufficient data, insufficient data, and fragmentary data. The cases represent different related fields: market share data, economic \\ growth data, and R\&D output data. The results show that the proposed grey-logistic method can effectively carry out the \\ population growth analysis.
}

\section{Introduction}

The development of any population will be restricted by its own growth ability and resource environment. Almost all species will obey the law of life cycle. Similarly, the development of the population in social economic system should not be an unlimited growth. If a social economic system is regarded as an ecosystem, the species in the social economic system can be regarded as the population in the ecosystem. The population dynamics model mainly focuses on the change of population quantity, and its change law is based on the nonlinear growth law of biological population quantity. Many species in nature grow nonlinearly, and such phenomenon is also very common. The competition and coordination mechanism within the population is also an important factor. This setting is based on the principle of intraspecies competition of biological population. There is competition in the natural biological population. The more populations there are, the more competitive it will be. Therefore, this mechanism should also become an important component of the growth model of the population.

The logistic growth function was first proposed by the Belgian scholar Verhulst in 1838 [1]. He used the logistic function to construct a growth curve, and after that, the curve had been used to conduct demographic research until the end of the nineteenth century. The British statistician Cox proposed the logistic regression model in 1958 [2]. This model's advantage in not making too many requirements in terms of normality, homogeneity of variance, etc., and the interpretability of coefficients has enabled logistic regression models to have been extensively adopted in many fields such as medicine and social surveys. The logistic regression model has been widely used for many years in the past. For example, it has already been used in the study of infectious diseases since its origin. As an effective data processing method, logistic regression analysis has a wide range of applications in many fields, such as biomedicine, criminology, ecological engineering, health, linguistics and wildlife zoology, and biology. Logistic regression model has made similar achievements in statistics. While studying the application of the logistic function and the logistic distribution in various fields, theoretical research on the estimation of the parameters of the logistic distribution and the test of the goodness of fit of the distribution has also achieved a series of important results. For example, literatures [3-6] give a variety of relationships between the logistic 
distribution and the extreme value distribution. Previous studies $[7,8]$ successively give the relationships between the logistic distribution and the exponential distribution. The achievement of these theoretical research has greatly promoted the study of theoretical issues such as the estimation of the parameters of the logistic distribution and the goodness-of-fit test of the distribution. Literatures $[9,10]$ give the best linear unbiased estimation of two parameters based on the complete data and the end data. Literature [11] analyzes the estimation problem based on logistic distribution parameters.

The current research mainly focuses on how to improve the validity and practicability of the logistic model by improving statistical estimation methods, and there are few researches on the preprocessing of raw data. Due to the insufficient amount of original data, measurement errors, missing data, and other problems, scholars call such data systems a grey system.

In terms of data characteristics, social economic system is also a grey system. Grey system theory is a method to study and solve a certain system with uncertain information, that is, grey system. Grey system is a system between black system and white system, which contains both known and unknown information. The information contained in grey system is called grey information. In the abstract systems of society, economy, and ecology, scholars should extend the viewpoints and methods of general cybernetics, system theory, and information theory to those abstract systems and make reasonable explanations.

The contents and arrangements of the research are as follows: (1) a compound grey transformation system based on $\operatorname{GM}(1,1)$ forecast model is constructed to transform the original data; (2) growth mechanism in population is analyzed by the model of grey-logistic system; (3) three cases are analyzed to illustrate the advantages of the composite forecast model. Highlights of the paper are as follows: (1) an improved grey-logistic forecast model is provided; (2) an analysis of population growth mechanism based on greylogistic model is made.

\section{Literature Review}

Grey system theory establishes a set of theories and methods to solve the related problems of incomplete information system, which has great development potential in practical applications. The grey system theory is a new theory that takes the grey system as the research object and uses a specific method to describe and control the grey system. In fact, the theory is to study the whitening problem of grey system, that is, to study how to use the existing information to predict the unknown information in the future systematically. The essence is to use the idea and method of grey system theory to quantify the abstract phenomenon, analyze the relevant data, and make quantitative forecast and control for the future, so as to complete the system analysis. GM (1, 1) model is widely used in grey system theory.

Grey system theory is a method to study and solve a certain system with uncertain information, that is, a grey system. GM $(1,1)$ model is widely used in grey system theory. GM $(1,1)$ model, proposed by Deng [12], has been widely applied in many fields [13]. GM $(1,1)$ model has been applied as a forecast approach in various fields, including wafer fabrication [14], optoelectronics industry forecasting [15], electricity forecasting [16], integrated circuit industry forecasting [17], product profit forecasting [18], and vehicle fatality risk forecasting [19]. However, as a single variable forecast model, traditional GM $(1,1)$ cannot be used to analyze the long-term relationship between the two variables.

In recent years, the research progress of GM $(1,1)$ model includes (1) the optimization of GM $(1,1)$ parameters based on initial condition [20], background [21], and accumulation order [22]; (2) the optimization of $\operatorname{GM}(1,1)$ structure [23]; and (3) the extension of GM $(1,1)$ modeling [24-28]. These above research results play an important role in improving the simulation and forecasting performance and expanding the application scope of GM $(1,1)$. Nevertheless, the classical GM $(1,1)$ model cannot be used in the ecological dynamics system. Considering the complicated relationship in natural, economic, and social ecosystems, it is necessary to analyze these relationships. Therefore, grey Lotka-Volterra model was applied to analyze the relationship in economic and social ecosystems [29]. After comparing forecast accuracy among the models proposed in this study, they have found that the proposed model has the best accuracy [30]. Mao et al. [31] established direct grey Lotka-Volterra model with adjustable background value. However, the current application of grey Lotka-Volterra model mainly discusses the relationship between the two populations and does not involve the analysis of the internal evolution of population. The application scenarios of logistic model are more abundant.

The grey system theory believes that although the objective system has complex appearance and disordered data, it has an overall function, so it must contain some internal law. The key lies in how to choose an appropriate method to dig it and use it. The impact of disturbance factors on the system behavior data sequence not only accelerates the development trend of the data or makes the oscillation amplitude of the data larger, but also slows down the development trend of the data or makes the oscillation amplitude of the data sequence smaller. In order to sort the interference of these impact factors, the buffer operator theory proposed by Professor Sifeng Liu can generate some sort of data sequencing, so as to weaken its randomness, show its regularity, and finally successfully eliminate the external impact interference and obtain a data sequence with the ability that reflects the changing laws of the system. As one of the methods to improve the accuracy of grey prediction model, data transformation technology is effective, and it also provides a new angle for the establishment of grey prediction model. In short, the data transformation technology is to perform a certain mapping transformation on the original data sequence under certain conditions and then establish a grey premodel, so as to achieve the purpose of improving the accuracy of simulation and prediction. The current research on data transformation technology is not in-depth. In general, the transformation should be in favor of improving smoothness. 
From the current research, data transformation technology is effective when used as a way to improve the prediction model. However, most documents only emphasize the improvement of smoothness in the transformation process, which is not helpful for improving the simulation and prediction accuracy of the grey prediction model. There have been documents showing with examples that the smoothness of the discrete data sequence after transformation has been greatly improved, but the prediction error has increased a lot. Therefore, the smoothness is only one of the factors that affect the simulation and prediction accuracy of the grey prediction model. In the specific transformation process, which criteria should be satisfied is worthy of research and discussion.

The grey combination forecasting model is extremely rich in content and has been widely used in practice. However, there are obvious deficiencies when it is rarely discussed in the current research literature about how to organically combine the grey forecasting model with other theoretical methods to find the combining point of the combined forecasting model. Grey system theory has its own research objects, the characteristics of which are "poor information" and "small samples," while the research objects of other theoretical methods are not necessarily characteristic of "poor information" and "small samples." Therefore, in the study of combined forecasting models, a combination point must be found.

\section{Methodology}

In this paper, the composite forecast model is constructed and compared with the traditional model. The methods used in this study are described below. Compared with the traditional GM $(1,1)$ model, this model can not only forecast, but also describe the dynamic mechanism of population growth. Compared with the traditional logistic model, this model adopts the grey transformation of the original data, which improves the forecast accuracy of logistic model.

3.1. Grey GM $(1,1)$ Model. Grey forecast is an approach based on the GM $(1,1)$ basic model to predict uncertain and incomplete information systems to determine the elements' future dynamic situation among a certain sequence of numbers.

As is shown in Figure 1, the processes of the GM $(1,1)$ model are shown as follows:

The original data series $X^{(0)}$ is

$$
X^{(0)}=\left\{x^{(0)}(1), x^{(0)}(2), \ldots, x^{(0)}(n)\right\}
$$

The accumulated generating operation $(\mathrm{AGO}) X^{(1)}(k)$ is

$$
\left\{\begin{array}{l}
X^{(1)}(k)=\sum_{i=1}^{k} X^{(0)}(i), \quad k=1,2,3, \ldots, n, \\
X^{(0)}=\left\{x^{(0)}(1), x^{(0)}(2), x^{(0)}(3), \ldots, x^{(0)}(n)\right\} .
\end{array}\right.
$$

The original form of GM $(1,1)$ model is

$$
X^{(0)}(k)+a X^{(1)}(k)=b .
$$

The parameter estimation based on the original form of the model and equation (3) is called the original difference grey model (ODGM) [9]. In the GM $(1,1)$ model, the parameters $a, b$ should be calculated first. Parameters $a, b$ can be calculated with the method of ordinary least square. The following model is an even GM $(1,1)$ system or even grey model (EGM). Practice shows that the simulation effect of the model is better, and it is also a commonly used grey model.

$$
\left\{\begin{array}{l}
X^{(0)}(k)+a z^{(1)}(k)=b, \\
z^{(1)}(k)=\frac{x^{(1)}(k)+x^{(1)}(k+1)}{2} \\
k=1,2,3, \ldots, n-1 .
\end{array}\right.
$$

After estimation of $a$ and $b$, the grey forecast equation can be solved as follows:

$$
\widehat{X}^{(1)}(k+1)=\left[X^{(0)}(1)-\frac{b}{a}\right] e^{-a k}+\frac{b}{a} .
$$

The following can be obtained based on inverse accumulated generating operation (IAGO)

$$
\begin{aligned}
& X^{(0)}(k+1)=X^{(1)}(k+1)-X^{(1)}(k)=\left[X^{(0)}(1)-\frac{b}{a}\right] e^{-a k}\left(1-e^{a}\right), \quad k=1,2,3, \ldots, n-1 . \\
& g(t)=\frac{\mathrm{d} N(t)}{\mathrm{d} t}=\alpha N\left(1-\frac{N}{K}\right),
\end{aligned}
$$

3.2. Logistic Model. The logistic function is a common sigmoid function. It was named by the mathematical biologist Velühle in 1844 when he was studying population growth. Logistic curve is often used to describe the population growth model under certain limited conditions. At the beginning, the curve grows roughly exponentially, at the later stage, it becomes saturated, and the growth slows down, and finally, the growth stops when it reaches full saturation.

As shown in Figure 2, the paper constructed internal dynamic system of population based on the Logistic model. $g(t)$ is the growth rate in phase $t . N(t)$ is population size of period $t . K$ is the maximum population scale. $\alpha$ is the intrinsic growth rate. $(1-(N / K))$ is the retardation of growth.

The measurement model is as follows.

Because $\mathrm{d} N(t) \approx \Delta N(t), \quad \Delta N(t)=N(t)-N(t-1)$, $d t \approx \Delta t=t-(t-1)=1$.

$$
\text { So : } g(t) \approx \Delta N(t)=\gamma_{1} N(t-1)+\gamma_{2} N^{2}(t-1) \text {. }
$$




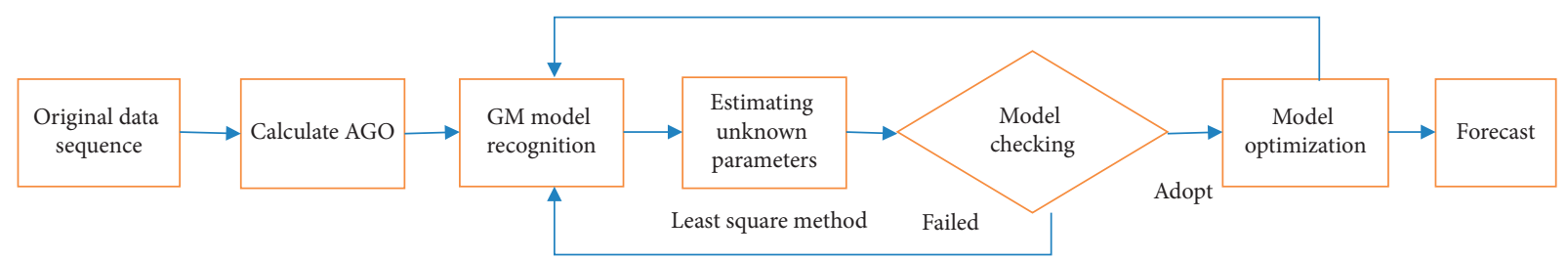

Figure 1: The GM $(1,1)$ model flow chart.

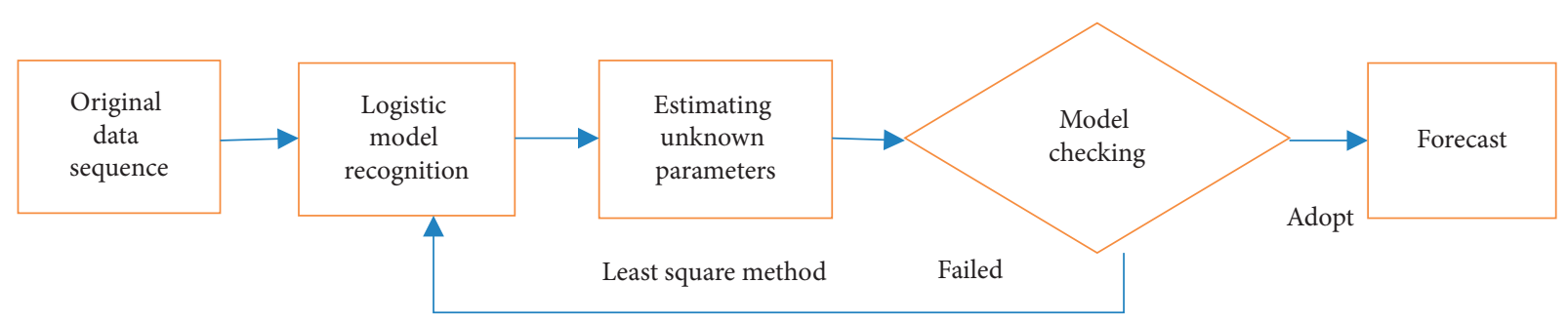

Figure 2: The Logistic model flow chart.

Through the regression processing of the original data, the relevant parameters can be obtained, and the obtained parameters can be used for forecast.

Among them, $\gamma_{1}=\alpha$, usually $\gamma_{1}>0$. It usually represents the synergy within a population. $\gamma_{2}=-(\alpha / K)$, usually, $\gamma_{2}<0$. It refers to the competition effect within a population. It is called internal competition coefficient or population density inhibition coefficient.

3.3. Compound Forecast Model: Grey-Logistic Model. Grey-logistic model is the combination of grey model and logistic model.

As shown in Figure 3, the paper constructed grey-logistic model based on the logistic model and GM $(1,1)$ model. The new information priority principle is one of the basic principles of grey system. Many scholars use this principle to optimize the grey GM $(1,1)$ model, but the priority of new information needs theoretical proof. Because the priority of the latest information in the traditional grey $\operatorname{GM}(1,1)$ forecast model cannot be compared intuitively, this paper constructs a composite GM $(1,1)$ grey data model from the perspective of making full use of the original data. The grey model is used to transform the observation data in order to obtain more abundant research information. The improved grey forecast model has shortcomings in terms of forecast accuracy, and its disadvantages will become more obvious as the amount of data increases, although it has great advantages dealing with data sets of uncertain factors. The logistic forecast is advantageous in its higher accuracy in fitting the data group with smaller fluctuations. In order to obtain a data set that can handle uncertain factors while ensuring accuracy requirements, a composite model of grey forecast and logistic forecast will be established here.

3.4. Comparison Model: Autoregression Moving Average (ARMA) Model. The autoregression moving average (ARMA) model is also called the Box-Jenkins model. The
ARMA model includes the autoregression part and the moving average part [32].

As shown in Figure 4, the autoregression moving average process can be expressed as $\operatorname{ARMA}(p, q)$.

The specific expressions are as follows:

$$
\begin{aligned}
X_{t}= & c+\eta_{1} X_{t-1}+\eta_{2} X_{t-2}+\cdots+\eta_{p} X_{t-p} \\
& +\varepsilon_{t}-\theta_{1} \varepsilon_{t-1}-\theta_{2} \varepsilon_{t-2}-\cdots-\theta_{q} \varepsilon_{t-q}
\end{aligned}
$$

where $\varepsilon t$ is the white noise, $c$ is a constant, $\eta_{1}, \eta_{2}, \ldots, \eta_{p}$ are the coefficients of the autoregressive model, and $\theta_{1}, \theta_{2}, \ldots, \theta_{q}$ are the coefficients of moving average model.

(1) Test the stability of the data with Dickey-Fuller Test (ADF). (2) Identify the model and the values of $p, q$ with autocorrelation function (ACF) and partial autocorrelation function (PACF). (3) Estimate and test the parameters of the model and calculate the AIC and SC values of the model. (4) Perform residual analysis by Box-Jenkins Q test. (5) Do prediction.

3.5. Prediction Accuracy Evaluation of Different Models. In this paper, the quality of reproduction will be measured by forecasting error and information fidelity of different forecast systems. The absolute percentage error (APE) and mean absolute percentage error (MAPE) index are used to evaluate the effect of grey transformation, among them

$$
\mathrm{MAPE}=\frac{1}{n} \sum_{k=1}^{n}\left|\frac{A_{k}-F_{k}}{A_{k}}\right| \times 100 \% .
$$

$A_{k}$ is the actual value, and $F_{k}$ is the forecasted value.

This paper will use entropy to describe the information fidelity of different forecast systems. The entropy value is used to measure the degree of chaos within the system. When the entropy value is larger, the system is more chaotic. Let $\langle U, \pi\rangle$ be an approximation space, where partition $\pi$ consists of blocks $U_{i}, 1 \leq i \leq k$, each of which has cardinality 


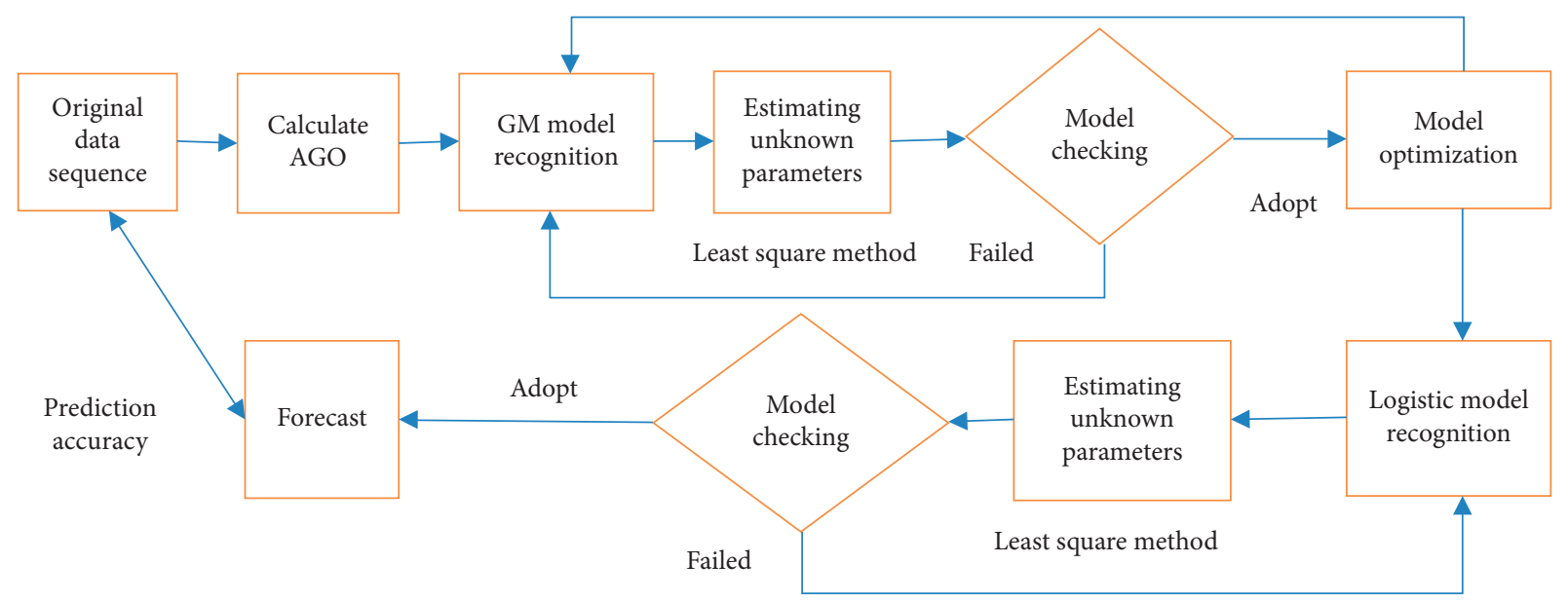

Figure 3: The grey-logistic model flow chart.

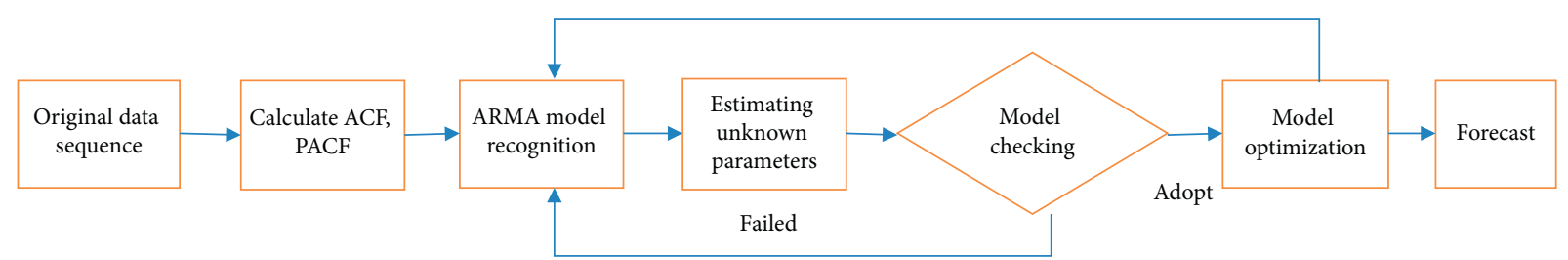

FIgURE 4: The ARMA method flow chart.

$n_{i}$. The information entropy $H(\pi)$ of partition $\pi$ is defined by [33]

$$
H(\pi)=-\sum_{i=1}^{k} \frac{n_{i}}{n} \log \frac{n_{i}}{n}, \quad \text { where } n=\sum_{i=1}^{k} n_{i} .
$$

The evaluation standard of information entropy is that the closer the entropy of forecast data is to that of the original data, the better. At the same time, the lower the entropy in different forecast systems, the better.

\section{Empirical Analysis}

4.1. Case 1: Forecast of FAW Volkswagen's Auto Sales Market Share. As a large-scale passenger car production enterprise, FAW Volkswagen Automotive Co., Ltd. (hereinafter referred to as FAW Volkswagen), established on February 6, 1991, and jointly operated by China FAW Co., Ltd., Volkswagen AG, Audi AG, and Volkswagen (China) Investment Co., Ltd, is China's first modern passenger car production enterprise built on an economic scale. After nearly 30 years of development, FAW Volkswagen's production capacity layout has covered Changchun in the northeast, Chengdu in the southwest, Foshan in the South, Qingdao in the East, and Tianjin in the North of China. It has eight professional production plants, namely, the first plant, the second plant, the third plant, the fourth plant, the fifth plant, the sixth plant, the powertrain division, and the stamping center. From one brand and one product at the beginning of the establishment of the factory, it has developed into one with more than 20 products under the three major brands of Audi, Volkswagen, and Jetta. FAW Volkswagen has occupied the No. 1 position in the passenger car market in China throughout the years (source: http:// www.faw-vw.com/).

As is shown in Table 1 , the original data in Case 1 is relatively sufficient. In this case, the grey GM $(1,1)$ model and the grey-logistic model have better forecast effects.

As shown in Table 2, the grey-logistic model in this case has the best forecast effect. The MAPE of the forecasted value of the grey-logistic model is $10.05 \%$, which is significantly lower than the $10.17 \%$ of the traditional GM $(1,1)$ model, also far lower than the $21.97 \%$ of the traditional logistic model. The MAPE of the traditional logistic model is less than $10 \%$ in the first ten forecast periods, and the model estimation effect is still relatively good. However, as the forecast period increases, the accuracy of the traditional logistic model is decreasing. This shows that the traditional logistic model is not suitable for long-term forecast. At the same time, because the original data is greyed in advance, the grey-logistic model overcomes the shortcomings of the traditional model and improves the accuracy of long-term forecast.

The $H(\pi)$ of raw data is 1.553 . Comparing the $H(\pi)$ values of the three forecast models and the original data from the perspective of information entropy, we can find that the difference between them is extremely small. The increase or decrease of entropy brought by the forecast model to the original system can be ignored. The forecast model has better information fidelity to the original data.

The model can be used not only to predict data, but also to describe the dynamic mechanism of system growth. The 
TABLE 1: Raw data and forecast data of FAW Volkswagen's market share.

\begin{tabular}{|c|c|c|c|c|c|c|c|c|c|}
\hline $\begin{array}{l}\text { Time } \\
\text { (month/year) }\end{array}$ & $\begin{array}{l}\text { Raw } \\
\text { data } \\
(\%)\end{array}$ & $\begin{array}{c}\text { Grey } \\
\text { forecast } \\
(\%)\end{array}$ & $\begin{array}{l}\text { Logistic } \\
\text { forecast } \\
\quad \%)\end{array}$ & $\begin{array}{c}\text { Grey- } \\
\text { logistic } \\
\text { forecast }(\%)\end{array}$ & $\begin{array}{c}\text { Time } \\
\text { (month/year) }\end{array}$ & $\begin{array}{l}\text { Raw } \\
\text { data } \\
(\%)\end{array}$ & $\begin{array}{c}\text { Grey } \\
\text { forecast } \\
(\%)\end{array}$ & $\begin{array}{l}\text { Logistic } \\
\text { forecast } \\
\quad \%)\end{array}$ & Grey-logistic forecast (\%) \\
\hline $2 / 18$ & 5.41 & 5.87 & 6.24 & 5.87 & $8 / 19$ & 7.42 & 6.27 & 4.77 & 6.28 \\
\hline $3 / 18$ & 5.68 & 5.93 & 6.14 & 5.89 & $9 / 19$ & 7.95 & 6.29 & 4.71 & 6.30 \\
\hline $4 / 18$ & 5.38 & 5.95 & 6.03 & 5.91 & $10 / 19$ & 7.60 & 6.31 & 4.65 & 6.32 \\
\hline $5 / 18$ & 5.61 & 5.97 & 5.94 & 5.94 & $11 / 19$ & 7.73 & 6.33 & 4.59 & 6.34 \\
\hline $6 / 18$ & 5.82 & 5.99 & 5.84 & 5.96 & $12 / 19$ & 5.80 & 6.35 & 4.53 & 6.36 \\
\hline $7 / 18$ & 6.67 & 6.01 & 5.75 & 5.99 & $1 / 20$ & 7.09 & 6.37 & 4.48 & 6.38 \\
\hline $8 / 18$ & 7.12 & 6.03 & 5.66 & 6.01 & $2 / 20$ & 4.07 & 6.39 & 4.42 & 6.40 \\
\hline $9 / 18$ & 6.31 & 6.05 & 5.57 & 6.03 & $3 / 20$ & 6.65 & 6.41 & 4.37 & 6.42 \\
\hline $10 / 18$ & 5.68 & 6.07 & 5.49 & 6.06 & $4 / 20$ & 5.71 & 6.43 & 4.32 & 6.44 \\
\hline $11 / 18$ & 5.42 & 6.09 & 5.41 & 6.08 & $5 / 20$ & 6.34 & 6.45 & 4.27 & 6.46 \\
\hline $12 / 18$ & 5.65 & 6.11 & 5.33 & 6.10 & $6 / 20$ & 6.22 & 6.47 & 4.22 & 6.48 \\
\hline $1 / 19$ & 5.26 & 6.13 & 5.25 & 6.13 & $7 / 20$ & 5.59 & 6.49 & 4.17 & 6.50 \\
\hline $2 / 19$ & 6.39 & 6.15 & 5.18 & 6.15 & $8 / 20$ & 6.49 & 6.51 & 4.12 & 6.52 \\
\hline $3 / 19$ & 5.69 & 6.17 & 5.10 & 6.17 & $9 / 20$ & 6.88 & 6.53 & 4.07 & 6.54 \\
\hline $4 / 19$ & 6.96 & 6.19 & 5.03 & 6.19 & $10 / 20$ & 6.94 & 6.55 & 4.03 & 6.56 \\
\hline $5 / 19$ & 6.06 & 6.21 & 4.96 & 6.21 & $11 / 20$ & 6.96 & 6.57 & 3.98 & 6.57 \\
\hline $6 / 19$ & 5.83 & 6.23 & 4.90 & 6.24 & $12 / 20$ & 5.66 & 6.60 & 3.94 & 6.59 \\
\hline $7 / 19$ & 6.74 & 6.25 & 4.83 & 6.26 & $1 / 21$ & 6.55 & 6.62 & 3.90 & 6.61 \\
\hline
\end{tabular}

TABLE 2: Error of estimation model: APE (MAPE, entropy).

\begin{tabular}{|c|c|c|c|c|c|c|c|}
\hline $\begin{array}{l}\text { Time } \\
\text { (month/year) }\end{array}$ & $\begin{array}{c}\text { Grey } \\
\text { forecast }(\%)\end{array}$ & $\begin{array}{c}\text { Logistic } \\
\text { forecast (\%) }\end{array}$ & $\begin{array}{l}\text { Grey-logistic } \\
\text { forecast }(\%)\end{array}$ & $\begin{array}{c}\text { Time } \\
\text { (month/year) }\end{array}$ & $\begin{array}{c}\text { Grey } \\
\text { forecast }(\%)\end{array}$ & Logistic forecast (\%) & Grey-logistic forecast (\%) \\
\hline $2 / 18$ & 8.41 & 6.42 & 6.04 & $9 / 19$ & 20.93 & 25.12 & 20.75 \\
\hline $3 / 18$ & 4.47 & 3.41 & 3.70 & $10 / 19$ & 17.02 & 26.31 & 16.83 \\
\hline $4 / 18$ & 10.65 & 1.37 & 9.93 & $11 / 19$ & 18.16 & 27.48 & 17.96 \\
\hline $5 / 18$ & 6.46 & 0.60 & 5.86 & $12 / 19$ & 9.42 & 28.61 & 9.69 \\
\hline $6 / 18$ & 2.95 & 2.51 & 2.45 & $1 / 20$ & 10.20 & 29.71 & 9.98 \\
\hline $7 / 18$ & 9.89 & 4.36 & 10.25 & $2 / 20$ & 56.94 & 30.79 & 57.31 \\
\hline $8 / 18$ & 15.31 & 6.15 & 15.59 & $3 / 20$ & 3.64 & 31.84 & 3.42 \\
\hline $9 / 18$ & 4.13 & 7.88 & 4.38 & $4 / 20$ & 12.58 & 32.86 & 12.82 \\
\hline $10 / 18$ & 6.84 & 9.56 & 6.64 & $5 / 20$ & 1.72 & 33.86 & 1.92 \\
\hline $11 / 18$ & 12.33 & 11.19 & 12.18 & $6 / 20$ & 4.01 & 34.84 & 4.20 \\
\hline $12 / 18$ & 8.10 & 12.77 & 8.02 & $7 / 20$ & 16.11 & 35.79 & 16.28 \\
\hline $1 / 19$ & 16.49 & 14.31 & 16.46 & $8 / 20$ & 0.33 & 36.72 & 0.45 \\
\hline $2 / 19$ & 3.81 & 15.80 & 3.78 & $9 / 20$ & 5.06 & 37.63 & 4.98 \\
\hline $3 / 19$ & 8.37 & 17.24 & 8.45 & $10 / 20$ & 5.58 & 38.52 & 5.53 \\
\hline $4 / 19$ & 11.12 & 18.65 & 11.02 & $11 / 20$ & 5.55 & 39.39 & 5.54 \\
\hline $5 / 19$ & 2.41 & 20.02 & 2.55 & $12 / 20$ & 16.52 & 40.23 & 16.48 \\
\hline $6 / 19$ & 6.79 & 21.34 & 6.97 & $1 / 21$ & 1.01 & 41.06 & 0.92 \\
\hline $7 / 19$ & 7.33 & 22.64 & 7.15 & MAPE & 10.17 & 21.97 & 10.05 \\
\hline $8 / 19$ & 15.55 & 23.90 & 15.38 & $H(\pi)$ & 1.556 & 1.552 & 1.556 \\
\hline
\end{tabular}

(Market share data source: http://xl.16888.com/f/57420/).

logistic measurement model based on grey transformation is as follows:

$$
\Delta N(t)=0.016 N(t-1)+(-0.205) N^{2}(t-1) .
$$

Through the regression processing of the grey transformation data, the relevant parameters can be obtained, and the obtained parameters can be used for forecast. Among them, $\alpha=0.016$, which represents the synergy within the population. $\gamma_{2}=-\alpha / K=-0.205$, usually, $\gamma_{2}<0$. It refers to the competition effect within a population. It is called internal competition coefficient or population density inhibition coefficient. In this case, the internal synergy and competition are significant. This shows that FAW-VW has a significant population growth effect within its products. In this paper, the least square method is used to estimate the model parameters, and the quality of the model estimation is mainly reflected by the prediction error. For the habit of time series research, this paper gives the relevant statistical test quantity for reference.

As shown in Table 3, the data of this case shows that the grey-logistic model has better statistical test data than logistic model. 
TABLE 3: Statistics of model parameter estimation for case 1.

\begin{tabular}{lccccc}
\hline Model & Variable & Coefficients & Std. error & $t$ stat & $P$-value \\
\hline \multirow{2}{*}{ Grey } & $b$ & 0.059 & 0.003 & 21.436 & -1.569 \\
\hline \multirow{2}{*}{ Logistic } & $a$ & -0.003 & 0.002 & 4.009 & 0.126 \\
& $\gamma_{1}$ & 0.736 & 0.184 & -4.122 & $<0.001$ \\
\multirow{2}{*}{ Grey-logistic } & $\gamma_{2}$ & -11.586 & 2.810 & 0.001 \\
& $\gamma_{1}$ & 0.016 & 0.007 & -1.884 & 0.023 \\
& $\gamma_{2}$ & -0.205 & 0.109 & 0.068 \\
\hline
\end{tabular}

4.2. Case 2: GDP in Jiangsu Province. Case 2, which uses the GDP data of Jiangsu Province as the object, analyzes the growth problem of an economic system. The selected amount of data is relatively small to test the forecast accuracy under the condition of small data. Jiangsu Province is a strong economic province in China. In 2020, the total economic volume of this province exceeded 10 trillion yuan. According to the preliminary calculations, the annual GDP of the province has reached 10,271.19 billion yuan, an increase of $3.7 \%$ over the previous year. Among them, the added value of the primary industry was 453.67 billion yuan, an increase of $1.7 \%$; the added value of the secondary industry was $4,422.64$ billion yuan, an increase of $3.7 \%$; the added value of the tertiary industry was 5,395.58 billion yuan, an increase of $3.8 \%$. It was estimated that the province's per capita GDP was 122,000 yuan, and the total labor productivity was 216,000 yuan per person. The adjustment of the industrial structure was accelerated. The ratio of the added value of the three industries throughout the year was adjusted to $4.4: 43.1: 52.5$, and the ratio of the added value of the service industry to GDP increased by $1.0 \%$ over the previous year. The economic vitality has increased. The nonpublic sector of the economy realized an added value of $7,693.65$ billion yuan throughout the year, accounting for $74.9 \%$ of GDP, an increase of $0.5 \%$ over the previous year; the value added of the private and individual economy accounted for $52.5 \%$ of GDP, and the value of private economy accounted for up to $56.8 \%$ of GDP. By the end of the year, there were 3.334 million private enterprises registered in the industrial and commercial sector, among which 518,000 were newly registered in 2020; at the same time, there were 8.556 million self-employed entrepreneurs, among which 2.077 million were newly registered in 2020 . With coordinated development of the region being vigorously promoted, the Yangtze River urban agglomeration has made a $79.2 \%$ contribution to the economic growth of the province; the contribution rate of the coastal economic belt to the economic growth of the province has reached $18.7 \%$ (resource: http://tj.jiangsu.gov.cn/art/2021/3/10/ art_4031_9698925.html).

As shown in Table 4, the MAPE of the forecast data of the grey-logistic model is only $2.57 \%$, and the grey-logistic model has the best predictive simulation effect. Under the condition of insufficient original data, the forecast effect of traditional logistic model is the worst. This case shows that the traditional GM $(1,1)$ grey prediction model and logistic model can be composite to deal with small data problems to achieve good results. From the perspective of entropy, the difference between the forecast data of several models and the actual data is extremely small. Several models forecast data with high information fidelity.

The logistic measurement model of GDP in Jiangsu is

$$
\Delta N(t)=0.103 N(t-1)+\left(-4.429 \times 10^{-20}\right) N^{2}(t-1) \text {. }
$$

Among them, $\alpha=0.103$, which represents the synergy within the population. Intrinsic growth rate of GDP in Jiangsu Province is $10.3 \% . \gamma_{2} \approx 0$, which means that the internal competition coefficient is close to zero. In this case, the internal synergy is significant. This shows that GDP in Jiangsu Province has a significant synergistic effect in economic growth. For the habit of time series research, this case gives the relevant statistical test quantity for reference. At the same time, it should be pointed out that the reference value of regression statistics under the condition of small amount of data or missing data is not large. The value of grey method is that it can better deal with the condition of lack of data and less data.

As shown in Table 5, the data of this case shows that grey-logistic model has better statistical test data than logistic model.

4.3. Case 3: Patent Grants in Jiangsu Province. Case 3 analyzes a growth problem of $\mathrm{R} \& \mathrm{D}$ output. In this case, the object of analysis is the data of patent grants in Jiangsu Province, a major and strong science and technology province in China. The data in this case is characteristic of sufficient missing of original data. This data system is a typical grey system.

In 2020, the number of patent applications and authorizations in Jiangsu Province reached 752,000 and 499,000, respectively, among which 189,000 were invention patent applications, an increase of $9.5 \%$ over the previous year; the number of invention patents was 46,000, an increase of $15.9 \%$. The province's enterprises have applied for 612,000 patents. The number of invention patents owned by ten thousand people reached 36.1, an increase of 6 over the same period last year; the contribution rate of scientific and technological progress was $65.1 \%$, an increase of $0.9 \%$ over the previous year. A total of 57,000 technical contracts of various types were signed throughout the year, and the transaction volume of technical contracts reached 233.58 billion yuan, an increase of $39.4 \%$ over the previous year. In the whole year, there were 922 crowd-creative enterprises on and above the provincial level. 
TABle 4: GDP in Jiangsu Province (unit: 100 million yuan), forecast value, and forecast accuracy evaluation.

\begin{tabular}{|c|c|c|c|c|c|c|c|}
\hline Year & Actual data & Grey forecast & APE (\%) & Logistic forecast & APE (\%) & Grey-logistic forecast & APE (\%) \\
\hline 2010 & 41384 & & & & & & \\
\hline 2011 & 48839 & 50063 & 2.50 & 48839 & 0.00 & & \\
\hline 2012 & 53702 & 55200 & 2.79 & 50053 & 6.79 & 53977 & 0.51 \\
\hline 2013 & 59349 & 60865 & 2.55 & 50259 & 15.32 & 59516 & 0.28 \\
\hline 2014 & 64831 & 67111 & 3.52 & 50294 & 22.42 & 65623 & 1.22 \\
\hline 2015 & 71256 & 73998 & 3.85 & 50300 & 29.41 & 72358 & 1.55 \\
\hline 2016 & 77351 & 81591 & 5.48 & 50301 & 34.97 & 79783 & 3.14 \\
\hline 2017 & 85870 & 89964 & 4.77 & 50301 & 41.42 & 87971 & 2.45 \\
\hline 2018 & 93208 & 99197 & 6.43 & 50301 & 46.03 & 96998 & 4.07 \\
\hline 2019 & 99632 & 109376 & 9.78 & 50301 & 49.51 & 106952 & 7.35 \\
\hline MAPE & & & 4.63 & & 27.32 & & 2.57 \\
\hline$H(\pi)$ & 0.894 & 0.892 & & 0.903 & & 0.892 & \\
\hline
\end{tabular}

TABle 5: Statistics of model parameter estimation for case 2.

\begin{tabular}{lccccc}
\hline Model & Variable & Coefficients & Std. error & $t$ stat & $P$-value \\
\hline \multirow{2}{*}{ Grey } & $b$ & 43620.848 & 627.808 & 69.481 & $<0.001$ \\
& $a$ & -0.098 & 0.002 & -53.366 & 0.001 \\
\hline \multirow{2}{*}{ Logistic } & $\gamma_{1}$ & 0.171 & 0.029 & 5.877 & 0.001 \\
& $\gamma_{2}$ & $<0.001$ & $<0.001$ & -2.712 & 0.030 \\
\hline \multirow{2}{*}{ Grey-logistic } & $\gamma_{1}$ & 0.103 & $<0.001$ & $2.916 * 10^{13}$ & $5.430 * 10^{7}$ \\
& $\gamma_{2}$ & $<0.001$ & $<0.001$ & $<0.001$ \\
\hline
\end{tabular}

The development of Jiangsu's high-tech industry has been accelerated. A total of 209 projects were organized and implemented for basic research projects of frontier leading technology, forward-looking industrial technology innovation projects, and major scientific and technological achievements transformation projects, with a provincial allocation of 1.05 billion yuan. In that year, there were 13,042 high-tech enterprises recognized; the rate of establishment of R\&D institutions for large- and medium-sized industrial enterprises and high-tech enterprises above designated size remained at around 90\%; the number of national-level R\&D institutions in enterprises reached 163, ranked among the best in China. The province has built 172 national industrial bases of high-tech characteristics.

Jiangsu Province has increased its investment in scientific research. The total social research and experimental development (R\&D) activities accounted for $2.85 \%$ of the regional GDP. The province has $890,000 \mathrm{R} \& \mathrm{D}$ staff and 105 academicians from the Chinese Academy of Sciences and the Chinese Academy of Engineering. Among various scientific research and technology development institutions, 435 independent research and development institutions are under government departments. 190 national and provincial key laboratories, 276 science and technology service platforms, 3,978 engineering technology research centers, and 126 enterprise academician workstations were built, and 1 comprehensive national technology innovation center was approved (source: http://tj.jiangsu.gov.cn/art/2021/3/10/ art_4031_9698925.html).

The characteristic of this case is missing the original data over a large amount of time period. This case uses simple interpolation to supplement the missing data and evenly distributes the difference between the two real pieces of data to each missing data period.

As shown in Table 6, the MAPE of the grey-logistic model forecast data is $16.58 \%$, and the grey-logistic model has the best forecast simulation effect. Under the condition that the original data is small and insufficient, the traditional logistic model has the worst forecast effect, and its forecast value basically has no reference value. This case shows that grey forecast model in the traditional GM $(1,1)$ compounded with logistic model can achieve the best results when dealing with the problem of missing information.

From the point of view of entropy, the difference in the entropy of the forecast data of several models is extremely small. Due to the sufficient missing of the original data in this case, the entropy value of the original data is significantly smaller than the entropy value of the data forecasted by several models. In this context, the information fidelity of the grey-logistic model is still relatively good.

The grey-logistic regression results of patent authorization in Jiangsu are

$$
\Delta N(t)=0.153 N(t-1)+\left(-3.817 \times 10^{-8}\right) N^{2}(t-1) .
$$

Among them, $\alpha=0.153$, which represents the synergy within the population. Intrinsic growth rate of patent authorization in Jiangsu Province is $15.3 \% ._{0}$, meaning that the internal competition coefficient is close to zero. In this case, the internal synergy is significant. This shows that patent 
TABLE 6: Patent grants in Jiangsu province, forecast value and forecast accuracy evaluation.

\begin{tabular}{|c|c|c|c|c|c|c|c|c|c|}
\hline Year & Actual data & Insert data & Complete series & Grey forecast & APE (\%) & Logistic forecast & APE (\%) & $\begin{array}{c}\text { Grey-logistic } \\
\text { forecast }\end{array}$ & APE (\%) \\
\hline 2000 & 6432 & & 6432 & & & & & & \\
\hline 2001 & & 19627 & 19627 & 56863 & 189.72 & 7417 & 62.21 & & \\
\hline 2002 & & 32822 & 32822 & 62940 & 91.76 & 16970 & 48.30 & 62940 & 91.76 \\
\hline 2003 & & 46017 & 46017 & 69665 & 51.39 & 35720 & 22.38 & 69665 & 51.39 \\
\hline 2004 & & 59212 & 59212 & 77110 & 30.23 & 64296 & 8.59 & 77110 & 30.23 \\
\hline 2005 & & 72407 & 72407 & 85350 & 17.88 & 103317 & 42.69 & 85350 & 17.88 \\
\hline 2006 & & 85602 & 85602 & 94471 & 10.36 & 153369 & 79.16 & 94471 & 10.36 \\
\hline 2007 & & 98797 & 98797 & 104566 & 5.84 & 214983 & 117.60 & 104566 & 5.84 \\
\hline 2008 & & 111992 & 111992 & 115741 & 3.35 & 288599 & 157.70 & 115741 & 3.35 \\
\hline 2009 & & 125187 & 125187 & 128109 & 2.33 & 374527 & 199.17 & 128109 & 2.33 \\
\hline 2010 & 138382 & 138382 & 138382 & 141799 & 2.47 & 472891 & 241.73 & 141799 & 2.47 \\
\hline 2011 & & 160763 & 160763 & 156952 & 2.37 & 583557 & 262.99 & 156952 & 2.37 \\
\hline 2012 & & 183144 & 183144 & 173724 & 5.14 & 706052 & 285.52 & 173724 & 5.14 \\
\hline 2013 & & 205525 & 205525 & 192289 & 6.44 & 839445 & 308.44 & 192289 & 6.44 \\
\hline 2014 & & 227906 & 227906 & 212837 & 6.61 & 982215 & 330.97 & 212837 & 6.61 \\
\hline 2015 & 250290 & 250287 & 250290 & 235582 & 5.88 & 1132072 & 352.30 & 235582 & 5.88 \\
\hline 2016 & 231033 & & 231033 & 260757 & 12.87 & 1285741 & 456.52 & 260757 & 12.87 \\
\hline 2017 & 227187 & & 227187 & 288622 & 27.04 & 1438688 & 533.26 & 288622 & 27.04 \\
\hline 2018 & 306996 & & 306996 & 319465 & 4.06 & 1584785 & 416.22 & 319465 & 4.06 \\
\hline 2019 & 314395 & & 314395 & 353604 & 12.47 & 1715882 & 445.77 & 353604 & 12.47 \\
\hline MAPE & & & & & 25.70 & & 230.08 & & 16.58 \\
\hline$H(\pi)$ & 0.774 & 1.101 & 1.204 & 1.217 & & 1.094 & & 1.200 & \\
\hline
\end{tabular}

Table 7: Statistics of model parameter estimation for Case 3.

\begin{tabular}{lccccc}
\hline Model & Variable & Coefficients & Std. error & $t$ stat & $P$-value \\
\hline \multirow{2}{*}{ Grey } & $b$ & 53379.956 & 7743.017 & 6.894 & $<0.001$ \\
& $a$ & -0.102 & 0.006 & 0.001 \\
\hline \multirow{2}{*}{ Logistic } & $\gamma_{1}$ & 0.153 & 0.074 & 2.059 & 0.055 \\
& $\gamma_{2}$ & $<0.001$ & $<0.001$ & -0.933 & 0.364 \\
\hline \multirow{2}{*}{ Grey-logistic } & $\gamma_{1}$ & 0.107 & $<0.001$ & $4.975 * 1013$ & $<0.001$ \\
& $\gamma_{2}$ & $<0.001$ & $<0.001$ & 1.078 & 0.297 \\
\hline
\end{tabular}

authorization in Jiangsu Province has a significant synergistic effect. This case gives the relevant statistical test quantity for reference.

As shown in Table 7, the data of this case shows that grey-logistic model has better statistical test data than the logistic model.

4.4. Comparison of Forecasting Accuracy of Different Models. In order to further test the prediction accuracy of grey-logistic model, this paper compares the prediction results of grey-logistic model, grey prediction model, and logistic model with AR model and ARMA model commonly used in time series analysis. These models have their own characteristics. Grey prediction model is essentially a way of data transformation and smoothing. Logistic model is similar to AR model, which is a kind of autoregressive model. The grey-logistic regression model is based on the grey processing of data. Grey GM $(1,1)$ model, logistic model, and grey-logistic model can be presented in the form of time function, which is convenient for prediction and analysis. Grey-logistic model is similar to ARMA model, both of them are the modification of autoregressive model. This section uses the relevant data in case 1 for comparative analysis.

As shown in Table 8, the average forecast values of greylogistic model and ARMA model are almost the same. The prediction data's volatility (the standard deviation of the predicted value) of the models is less than that of the original data, and the volatility of the prediction data of ARMA model is the smallest.

As shown in Table 9, the prediction accuracy of a greylogistic forecast model is the highest, and the prediction accuracy of grey model ranks second. The prediction accuracy of the logistic forecast and AR forecast models is poor. The prediction accuracy of ARMA model is close to that of the grey-logistic forecast model. The data used in this section is a relatively rich data volume. When the amount of data is less and missing, the accuracy advantage of grey model and grey-logistic model will be more significant.

As shown in Figure 5, the accuracy trend of ARMA model is close to that of the grey-logistic forecast model. The graphs reflecting the accuracy trend of the two models are highly similar. This phenomenon is due to the similarity of the two models, and they are the improvement of 
TABLE 8: Forecasting results of different models.

\begin{tabular}{|c|c|c|c|c|c|c|}
\hline $\begin{array}{l}\text { Time (month/ } \\
\text { year) }\end{array}$ & $\begin{array}{l}\text { Actual data } \\
(\%)\end{array}$ & $\begin{array}{c}\text { Grey forecast } \\
(\%)\end{array}$ & $\begin{array}{c}\text { Logistic forecast } \\
(\%)\end{array}$ & $\begin{array}{c}\text { Grey-logistic forecast } \\
(\%)\end{array}$ & $\begin{array}{c}\mathrm{AR}(1) \text { forecast } \\
(\%)\end{array}$ & $\begin{array}{c}\text { ARMA }(1,1) \text { forecast } \\
(\%)\end{array}$ \\
\hline $1 / 18$ & 6.99 & & & & & \\
\hline $2 / 18$ & 5.41 & 5.87 & 6.24 & 5.87 & 7.89 & 6.46 \\
\hline $3 / 18$ & 5.68 & 5.93 & 6.14 & 5.89 & 7.53 & 6.37 \\
\hline $4 / 18$ & 5.38 & 5.95 & 6.03 & 5.91 & 7.59 & 6.32 \\
\hline $5 / 18$ & 5.61 & 5.97 & 5.94 & 5.94 & 7.52 & 6.30 \\
\hline $6 / 18$ & 5.82 & 5.99 & 5.84 & 5.96 & 7.57 & 6.29 \\
\hline $7 / 18$ & 6.67 & 6.01 & 5.75 & 5.99 & 7.62 & 6.28 \\
\hline $8 / 18$ & 7.12 & 6.03 & 5.66 & 6.01 & 7.81 & 6.28 \\
\hline $9 / 18$ & 6.31 & 6.05 & 5.57 & 6.03 & 7.92 & 6.27 \\
\hline $10 / 18$ & 5.68 & 6.07 & 5.49 & 6.06 & 7.73 & 6.27 \\
\hline $11 / 18$ & 5.42 & 6.09 & 5.41 & 6.08 & 7.59 & 6.27 \\
\hline $12 / 18$ & 5.65 & 6.11 & 5.33 & 6.10 & 7.53 & 6.27 \\
\hline $1 / 19$ & 5.26 & 6.13 & 5.25 & 6.13 & 7.58 & 6.27 \\
\hline $2 / 19$ & 6.39 & 6.15 & 5.18 & 6.15 & 7.49 & 6.27 \\
\hline $3 / 19$ & 5.69 & 6.17 & 5.10 & 6.17 & 7.75 & 6.27 \\
\hline $4 / 19$ & 6.96 & 6.19 & 5.03 & 6.19 & 7.59 & 6.27 \\
\hline $5 / 19$ & 6.06 & 6.21 & 4.96 & 6.21 & 7.88 & 6.27 \\
\hline $6 / 19$ & 5.83 & 6.23 & 4.90 & 6.24 & 7.68 & 6.27 \\
\hline $7 / 19$ & 6.74 & 6.25 & 4.83 & 6.26 & 7.62 & 6.27 \\
\hline $8 / 19$ & 7.42 & 6.27 & 4.77 & 6.28 & 7.83 & 6.27 \\
\hline $9 / 19$ & 7.95 & 6.29 & 4.71 & 6.30 & 7.98 & 6.27 \\
\hline $10 / 19$ & 7.60 & 6.31 & 4.65 & 6.32 & 8.10 & 6.27 \\
\hline $11 / 19$ & 7.73 & 6.33 & 4.59 & 6.34 & 8.03 & 6.27 \\
\hline $12 / 19$ & 5.80 & 6.35 & 4.53 & 6.36 & 8.05 & 6.27 \\
\hline $1 / 20$ & 7.09 & 6.37 & 4.48 & 6.38 & 7.62 & 6.27 \\
\hline $2 / 20$ & 4.07 & 6.39 & 4.42 & 6.40 & 7.91 & 6.27 \\
\hline $3 / 20$ & 6.65 & 6.41 & 4.37 & 6.42 & 7.22 & 6.27 \\
\hline $4 / 20$ & 5.71 & 6.43 & 4.32 & 6.44 & 7.81 & 6.27 \\
\hline $5 / 20$ & 6.34 & 6.45 & 4.27 & 6.46 & 7.60 & 6.27 \\
\hline $6 / 20$ & 6.22 & 6.47 & 4.22 & 6.48 & 7.74 & 6.27 \\
\hline $7 / 20$ & 5.59 & 6.49 & 4.17 & 6.50 & 7.71 & 6.27 \\
\hline $8 / 20$ & 6.49 & 6.51 & 4.12 & 6.52 & 7.57 & 6.27 \\
\hline $9 / 20$ & 6.88 & 6.53 & 4.07 & 6.54 & 7.77 & 6.27 \\
\hline $10 / 20$ & 6.94 & 6.55 & 4.03 & 6.56 & 7.86 & 6.27 \\
\hline $11 / 20$ & 6.96 & 6.57 & 3.98 & 6.57 & 7.88 & 6.27 \\
\hline $12 / 20$ & 5.66 & 6.60 & 3.94 & 6.59 & 7.88 & 6.27 \\
\hline $1 / 21$ & 6.55 & 6.62 & 3.90 & 6.61 & 7.58 & 6.27 \\
\hline Mean & 6.28 & 6.26 & 4.89 & 6.26 & 7.72 & 6.28 \\
\hline Std. deviation & 0.80 & 0.21 & 0.69 & 0.22 & 0.19 & 0.03 \\
\hline
\end{tabular}

TABLe 9: Absolute percentage error (APE) of different forecasting models.

\begin{tabular}{|c|c|c|c|c|c|}
\hline Time (month/year) & Grey forecast (\%) & Logistic forecast (\%) & Grey-logistic forecast (\%) & $\mathrm{AR}(1)$ forecast $(\%)$ & ARMA $(1,1)$ forecast $(\%)$ \\
\hline $2 / 18$ & 8.41 & 15.38 & 8.41 & 45.78 & 19.34 \\
\hline $3 / 18$ & 4.47 & 8.04 & 3.70 & 32.54 & 12.14 \\
\hline $4 / 18$ & 10.65 & 12.17 & 9.93 & 41.07 & 17.54 \\
\hline $5 / 18$ & 6.46 & 5.82 & 5.86 & 34.07 & 12.29 \\
\hline $6 / 18$ & 2.95 & 0.36 & 2.45 & 30.13 & 8.01 \\
\hline $7 / 18$ & 9.89 & 13.81 & 10.25 & 14.26 & 5.85 \\
\hline $8 / 18$ & 15.31 & 20.52 & 15.59 & 9.75 & 11.85 \\
\hline $9 / 18$ & 4.13 & 11.69 & 4.38 & 25.46 & 0.57 \\
\hline $10 / 18$ & 6.84 & 3.37 & 6.64 & 36.13 & 10.44 \\
\hline $11 / 18$ & 12.33 & 0.25 & 12.18 & 40.03 & 15.73 \\
\hline $12 / 18$ & 8.10 & 5.71 & 8.02 & 33.28 & 11.02 \\
\hline $1 / 19$ & 16.49 & 0.18 & 16.46 & 44.15 & 19.24 \\
\hline $2 / 19$ & 3.81 & 19.00 & 3.78 & 17.28 & 1.84 \\
\hline $3 / 19$ & 8.37 & 10.31 & 8.45 & 36.21 & 10.23 \\
\hline $4 / 19$ & 11.12 & 27.69 & 11.02 & 9.08 & 9.88 \\
\hline
\end{tabular}


TABle 9: Continued.

\begin{tabular}{|c|c|c|c|c|c|}
\hline Time (month/year) & Grey forecast (\%) & Logistic forecast (\%) & Grey-logistic forecast (\%) & $\mathrm{AR}(1)$ forecast (\%) & ARMA $(1,1)$ forecast $(\%)$ \\
\hline $5 / 19$ & 2.41 & 18.09 & 2.55 & 30.03 & 3.50 \\
\hline $6 / 19$ & 6.79 & 16.00 & 6.97 & 31.66 & 7.58 \\
\hline $7 / 19$ & 7.33 & 28.31 & 7.15 & 13.11 & 6.94 \\
\hline $8 / 19$ & 15.55 & 35.73 & 15.38 & 5.53 & 15.47 \\
\hline $9 / 19$ & 20.93 & 40.79 & 20.75 & 0.43 & 21.11 \\
\hline $10 / 19$ & 17.02 & 38.86 & 16.83 & 6.64 & 17.47 \\
\hline $11 / 19$ & 18.16 & 40.65 & 17.96 & 3.82 & 18.86 \\
\hline $12 / 19$ & 9.42 & 21.88 & 9.69 & 38.87 & 8.14 \\
\hline $1 / 20$ & 10.20 & 36.88 & 9.98 & 7.43 & 11.54 \\
\hline $2 / 20$ & 56.94 & 8.62 & 57.31 & 94.33 & 54.10 \\
\hline $3 / 20$ & 3.64 & 34.32 & 3.42 & 8.63 & 5.68 \\
\hline $4 / 20$ & 12.58 & 24.42 & 12.82 & 36.77 & 9.84 \\
\hline $5 / 20$ & 1.72 & 32.73 & 1.92 & 19.81 & 1.07 \\
\hline $6 / 20$ & 4.01 & 32.22 & 4.20 & 24.42 & 0.84 \\
\hline $7 / 20$ & 16.11 & 25.45 & 16.28 & 37.96 & 12.20 \\
\hline $8 / 20$ & 0.33 & 36.51 & 0.45 & 16.62 & 3.36 \\
\hline $9 / 20$ & 5.06 & 40.78 & 4.98 & 12.98 & 8.84 \\
\hline $10 / 20$ & 5.58 & 41.95 & 5.53 & 13.28 & 9.62 \\
\hline $11 / 20$ & 5.55 & 42.75 & 5.54 & 13.15 & 9.88 \\
\hline $12 / 20$ & 16.52 & 30.36 & 16.48 & 39.22 & 10.81 \\
\hline $1 / 21$ & 1.01 & 40.47 & 0.92 & 15.80 & 4.24 \\
\hline Mean & 10.17 & 22.84 & 10.12 & 25.55 & 11.31 \\
\hline
\end{tabular}

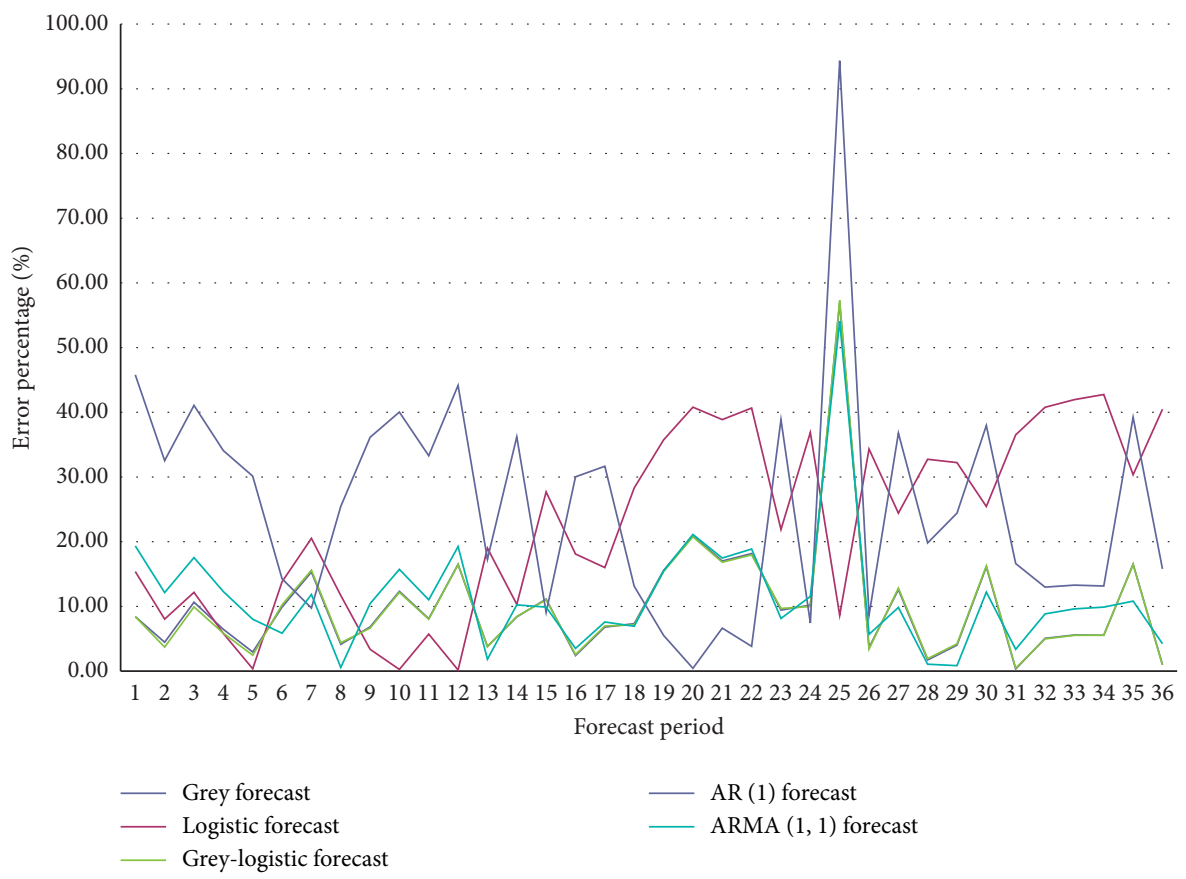

FIGURE 5: Comparison of forecasting errors of different models.

the autoregressive model. At the same time, the greylogistic system has more variety and more practical advantages. For example, (1) the grey-logistic model can be used to explain population dynamics. The grey-logistic model is an improvement of traditional population dynamics model, which is suitable for the research of ecology related fields. (2) It can be constructed as a generalized prediction model with time as the independent variable, which is extremely suitable for a wide range of prediction researches. (3) Considering the advantages 
of traditional grey model and logistic model, the application scenarios are more extensive than the ARMA model, especially in the case of insufficient and missing original data.

\section{Conclusion}

In order to improve the performance of $\mathrm{GM}(1,1)$ forecast and logistic forecast, a new approach to estimate the parameters of the socioeconomic system is presented. The composite grey-logistic model is proposed to estimate the parameters of the forecast model. Based on grey model and logistic model, this paper constructs grey-logistic model. Through the analysis of actual cases, the accuracy of greylogistic model is compared with that of the grey model, logistic model, AR model, and ARMA model. The empirical results indicate that the numerical aspects of the grey transformation effect of the composite grey-Logistic model's MAPE values are highly accurate. The results of entropy analysis show that the grey-logistic model can keep the information of the original data better. As compared with the GM $(1,1)$, the composite grey model used in this study offers more accurate forecast performance. Compared with the traditional logistic model, the new grey-logistic model used in this study offers more accurate forecast performance too. The original data after grey transformation can more truly reflect the actual situation of the social and economic system. The combination of grey transformation data and logistic model can mine the symbiotic relationship of population in a certain system; accordingly, this method is suitable when the data are limited for forecasting the relationship between competitive products. The empirical analysis fully demonstrates the effectiveness and adaptability of this method.

Compared with the traditional grey model [12-20], the compound grey-logistic model can well describe the dynamic mechanism of population growth. Grey-logistic model is more suitable for the research of social and economic ecosystem. Compared with the traditional logistic model [6-11], the grey-logistic model has higher accuracy. Compared with the grey Lotka-Volterra model [29-31], the grey-logistic model is more applicable. Compared with AR and ARMA models in time series analysis, the grey-logistic model has higher precision, and the grey-logistic model has ecological significance and can be used for the classification of systems.

The case analysis based on grey-logistic model fully demonstrates the applicability and accuracy of the method in different data types and research fields. Grey-logistic model can effectively deal with the problem of insufficient data and missing data and more fully explore the information carried by the original data. This model can be widely used in population dynamics analysis. When it is difficult to carry out regression analysis in the traditional logistic model, we can try to use grey-logistic model to deal with it. In the future, this method can be extended to the analysis of two population interactions and three population symbioses.

\section{Data Availability}

The experimental data used to support the findings of this study are included within the article.

\section{Conflicts of Interest}

The authors declare that they have no conflicts of interest.

\section{Acknowledgments}

This work was supported by the National Social Science Foundation of China (No. 20BGL203).

\section{References}

[1] P. F. Verhulst, "Notice sur la loi que la population suit dans son accroissement," Correspondence Mathematique et Physique (Ghent), vol. 10, pp. 113-121, 1838.

[2] D. R. Cox, "The regression analysis of binary sequences," Journal of the Royal Statistical Society: Series B (Methodological), vol. 20, no. 2, pp. 215-232, 1958.

[3] E. J. Gumbel, "Ranges and midranges," Annals of Mathematical Statistics, vol. 15, pp. 414-422, 1994.

[4] E. J. Gumbel and R. D. Keeney, "The extremal quotient," The Annals of Mathematical Statistics, vol. 21, no. 4, pp. 523-538, 1950.

[5] E. J. Gumbel and J. Pickands, "Probability tables for the extreme quotient," Annals of Mathematical Statistics, vol. 38, pp. 1441-1451, 1967.

[6] S. D. Dubey, "A new derivation of the logistic distribution," Naval Research Logistics Quarterly, vol. 16, no. 1, pp. 37-40, 1969.

[7] E. O. George and E. S. A. Mudholkar, "Characterization of the logistic by A sample median," Annals of the Institute of Statistical Mathematics, vol. 33, pp. 125-129, 1981.

[8] E. O. George and G. S. Mudholkar, "On the logistic and exponential laws," Sankhya, Set, A, vol. 44, pp. 291-293, 1982.

[9] E. X. Frank, "Logistic regression and boosting for labeled bags of instances," Lecture Notes in Computer Science, vol. 56, pp. 272-281, 2004.

[10] N. Balakrishnan, Handbook of the Logisitic Distribution, Marcel Dekker Inc, New York, NY, USA, 1992.

[11] N. Balakrisheaa, "Approximate MLES for the location scale parameters of the half-logistic distribution with type II rightcensoring," IEEE Transactions on Reliability, vol. 40, pp. 140-145, 1991.

[12] J. L. Deng, "Introduction Grey system theory," Journal of Grey System, vol. 1, no. 1, pp. 191-243, 1989.

[13] S. F. Liu and Y. Lin, Grey Systems: Theory and Practical Applications, Springer-Verlag London Ltd, London, UK, 2010.

[14] Y.-S. Chen and B.-Y. Chen, "Applying DEA, MPI, and grey model to explore the operation performance of the Taiwanese wafer fabrication industry," Technological Forecasting and Social Change, vol. 78, no. 3, pp. 536-546, 2011.

[15] C.-T. Lin and S.-Y. Yang, "Forecast of the output value of Taiwan's opto-electronics industry using the Grey forecasting model," Technological Forecasting and Social Change, vol. 70, no. 2, pp. 177-186, 2003.

[16] S.-C. Lee and L.-H. Shih, "Forecasting of electricity costs based on an enhanced gray-based learning model: a case study 
of renewable energy in Taiwan," Technological Forecasting and Social Change, vol. 78, no. 7, pp. 1242-1253, 2011.

[17] L.-C. Hsu, "Applying the Grey prediction model to the global integrated circuit industry," Technological Forecasting and Social Change, vol. 70, no. 6, pp. 563-574, 2003.

[18] Y.-Y. Tai, J.-Y. Lin, M.-S. Chen, and M.-C. Lin, "A grey decision and prediction model for investment in the core competitiveness of product development," Technological Forecasting and Social Change, vol. 78, no. 7, pp. 1254-1267, 2011.

[19] M. Mao and E. C. Chirwa, "Application of grey model GM(1, 1) to vehicle fatality risk estimation," Technological Forecasting and Social Change, vol. 73, no. 5, pp. 588-605, 2006.

[20] J. J. Wang, Y. G. Dang, J. Ye, N. Xu, and J. Wang, “An improved grey prediction model based on matrix representations of the optimized initial value," 7e Journal of Grey System, vol. 30, no. 3, pp. 143-156, 2018.

[21] B. Zeng and C. Li, "Improved multi-variable grey forecasting model with a dynamic background-value coefficient and its application," Computers \& Industrial Engineering, vol. 118, pp. 278-290, 2018.

[22] X. Ma, W. Wu, B. Zeng, Y. Wang, and X. Wu, "The conformable fractional grey system model," ISA Transactions, vol. 96, pp. 255-271, 2020.

[23] X. Ma, M. Xie, W. Wu, B. Zeng, Y. Wang, and X. Wu, "The novel fractional discrete multivariate grey system model and its applications," Applied Mathematical Modelling, vol. 70, pp. 402-424, 2019.

[24] J. Ye, Y. Dang, S. Ding, and Y. Yang, “A novel energy consumption forecasting model combining an optimized DGM $(1,1)$ model with interval grey numbers," Journal of Cleaner Production, vol. 229, pp. 256-267, 2019.

[25] B. Zeng, M. Tong, and X. Ma, "A new-structure grey Verhulst model: development and performance comparison," Applied Mathematical Modelling, vol. 81, pp. 522-537, 2020.

[26] Y.-H. Wu and H. Shen, "Grey-related least squares support vector machine optimization model and its application in predicting natural gas consumption demand," Journal of Computational and Applied Mathematics, vol. 338, pp. 212220, 2018.

[27] X. Ma, "A brief introduction to the grey machine learning," The Journal of Grey System, vol. 31, pp. 1-12, 2019.

[28] S. F. Liu, Y. J. Yang, and J. Forrest, Grey Data Analysis Methods, Models and Applications, Springer-Verlag, Berlin, Germany, 2016.

[29] L. Wu, S. Liu, and Y. Wang, "Grey Lotka-Volterra model and its application," Technological Forecasting and Social Change, vol. 79, no. 9, pp. 1720-1730, 2012.

[30] H.-T. Wang and T.-C. Wang, "Application of the grey LotkaVolterra model to forecast the diffusion and competition analysis of the TV and smartphone industries," Technological Forecasting and Social Change, vol. 106, pp. 37-44, 2016.

[31] S. H. Mao, M. Zhu, X. P. Wang, and X. P. Xiao, "Grey-LotkaVolterra model for the competition and cooperation between third-party online payment systems and online banking in China," Applied Soft Computing, vol. 95, no. 106501, 2020.

[32] G. E. P. Box and G. M. Jenkins, "Time series analysis: forecasting and control," Journal of Time, vol. 31, no. 4, p. 303, 2010.

[33] Y. Y. Yao, "Probabilistic approaches to rough sets," Expert Systems, vol. 20, no. 5, pp. 287-297, 2003. 